\title{
Article
}

\section{QSAR Analyses of Skin Penetration Enhancers}

\author{
Manisha lyer, Tao Zheng, A. J. Hopfinger, and Yufeng J. Tseng
}

J. Chem. Inf. Model., 2007, 47 (3), 1130-1149• DOI: 10.1021/ci700051e • Publication Date (Web): 02 May 2007

\section{Downloaded from http://pubs.acs.org on March 19, 2009}

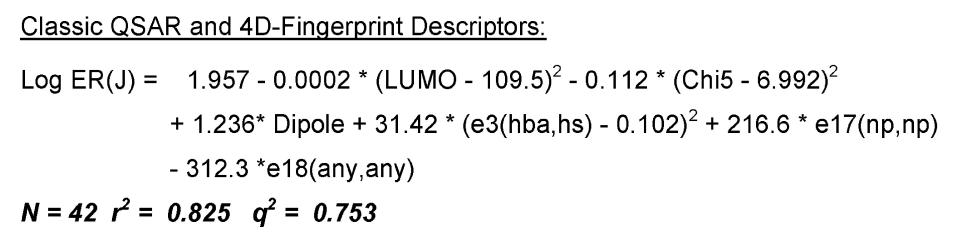

\section{More About This Article}

Additional resources and features associated with this article are available within the HTML version:

- $\quad$ Supporting Information

- $\quad$ Links to the 3 articles that cite this article, as of the time of this article download

- $\quad$ Access to high resolution figures

- $\quad$ Links to articles and content related to this article

- $\quad$ Copyright permission to reproduce figures and/or text from this article

\section{View the Full Text HTML}




\title{
QSAR Analyses of Skin Penetration Enhancers
}

\author{
Manisha Iyer ${ }^{\dagger}$ Tao Zheng, ${ }^{\ddagger}$ A. J. Hopfinger, ${ }^{\S, \|}$ and Yufeng J. Tseng*,L,\|
}

Division of Clinical Chemistry, Department of Pathology, Children's Hospital of Pittsburgh, 5834 Main Tower, 200 Lothrop Street, Pittsburgh, Pennsylvania 15213, The Chem21 Group, Inc., 1780 Wilson Drive, Lake Forest, Illinois 60045, College of Pharmacy, MSC09 5360, 1 University of New Mexico, Albuquerque, New Mexico 87131-0001, New Technology Department, Global Research \& Development, Avon Products, Inc., 1 Avon Place, Suffern, New York 10901-5605, and Graduate Institute of Biomedical Electronics and Bioinformatics, Department of Computer Science and Information Engineering, National Taiwan University, No. 1 Sec. 4, Roosevelt Road, Taipei, Taiwan 106

Received February 7, 2007

\begin{abstract}
QSAR models for four skin penetration enhancer data sets of $61,44,42$, and 17 compounds were constructed using classic QSAR descriptors and 4D-fingerprints. Three data sets involved skin penetration enhancement of hydrocortisone and hydrocortisone acetate. The other data set involved skin penetration enhancement of fluorouracil. The measure of penetration enhancement is the ratio of the net permeation of the penetrant with and without a common fixed concentration of enhancer. Significant QSAR models could be built using multidimensional linear regression fitting and genetic function model optimization for all four data sets when both classic and 4D-fingerprint descriptors were used in the trial descriptor pool. Reasonable QSAR models could be built when only 4D-fingerprint descriptors were employed, and no significant QSAR models could be built using only classic descriptors for two of the four data sets. Comparison analyses of the descriptor terms, and their respective regression coefficients, across the pairs of the best QSAR models of the four skin penetration enhancer data sets did not reveal any significant extent of similar terms. Overall, the QSAR models for the penetration-enhancer systems appear meaningfully different from one another, suggesting that there are distinct mechanisms of skin penetration enhancement that depend on the chemistry of both the enhancer and the penetrant.
\end{abstract}

\section{INTRODUCTION}

The targeted enhancement of the skin permeability to a particular drug or therapeutic agent represents an attractive means for systemic drug administration. Thus, the development of penetration enhancers as transport facilitators to compounds of low percutaneous absorption continues to be of high interest for delivery systems in both the cosmetic and pharmaceutical industries. The stratum corneum (SC) has been identified as the rate-limiting barrier in skin for the percutaneous absorption of drugs and other organics. The SC consists of dead cells surrounded by multilamellar lipid bilayer membranes. Small hydrophobic or nonpolar molecules can partition into the SC and then diffuse across the lipid bilayer membranes, the intercellular route. However, hydrophilic (polar) molecules can penetrate the SC only through the transcellular route or pre-existing aqueous pathways associated with sweat gland ducts and hair follicles. Many experimental studies ${ }^{1}$ have investigated the action of chemical enhancers, and the following enhancement mechanisms have been suggested: (1) interaction with intercellular lipids of the SC, resulting in disorganization of these highly ordered structures and thus enhancing the paracellular

* Corresponding author phone: 886-2-3366-4888 \#529; fax: 886-223628167; e-mail: yjtseng@csie.ntu.edu.tw.

Children's Hospital of Pittsburgh.

$\doteqdot$ Avon Products, Inc.

$\S 1$ University of New Mexico.

" The Chem21 Group, Inc.

${ }^{\perp}$ National Taiwan University. diffusivity through the SC; (2) interaction with intracellular proteins of the corneocytes to increase transcellular permeation; and (3) increasing partitioning of the drug into the SC. Although experiments have suggested multiple mechanisms of enhancer action, detailed molecular modeling studies based on the molecular structures of the chemical enhancers are needed to elucidate the mechanisms of enhancement and also to permit predict enhancement potency.

Some experimental studies of penetration enhancement have measured the lipophilicity [nonpolarity] of the enhancers and also investigated the corresponding relationships between enhancer nonpolarity and penetration enhancement potency. ${ }^{2}$ But in general there have been few molecular modeling and QSAR studies of skin penetration enhancers. One reason may be that each compound whose penetration is to be enhanced produces a distinct structure or an activity relationship for a given set of enhancers. Said another way, a QSAR developed for one drug for a given class of skin penetration enhancers may not apply to a second drug. Thus, a unique QSAR model may be needed for each drug and penetration enhancer data set, and generalizations from molecular modeling may be limited. However, it does appear that a common set of physicochemical properties governs the penetration enhancement of nonpolar drugs which are different from those of polar drugs. ${ }^{3}$

This paper reports the development of QSAR models and affiliated findings and conclusions for four different skin penetration enhancer data sets. Three of the data sets deal 
Table 1. Training Set 1: 61 Surfactant-like and/or Nonpolar Enhancers Using Hydrocortisone (HC) as a Master Reference Penetrator

\begin{tabular}{|c|c|c|c|}
\hline Code & Structure & $E R(J)$ & Reference \\
\hline REF-AZONE/HC-1 & & $\begin{array}{l}\text { (a) } 19.51 \\
\text { (b) } 42.20 \\
\text { (c) } 18.00\end{array}$ & $\begin{array}{l}\text { (a) Michniak et. al. Int. J. } \\
\text { Pharm. 1995, 116, 201- } \\
\text { 209; } \\
\text { (b) Michniak et. al. Int. J. } \\
\text { Pharm. 1998, 161, 169- } \\
178 ; \\
\text { (c) Fuhrman Jr. et. al. J. } \\
\text { Controlled Release 1997 } \\
45,199-206 .\end{array}$ \\
\hline SL-LACTAM-ACETIC-ESTERS/HC-1 & & 18.89 & $\begin{array}{l}\text { Michniak et. al. J. Pharm. } \\
\text { Sci. 1996, } 85 \text { (2), 150- } \\
154\end{array}$ \\
\hline SL-LACTAM-ACETIC-ESTERS/HC-2 & & 17.55 & $\begin{array}{l}\text { Michniak et. al. J. Pharm } \\
\text { Sci. 1996, } 85 \text { (2), 150- } \\
154\end{array}$ \\
\hline SL-LACTAM-ACETIC-ESTERS/HC-3 & & 38.22 & $\begin{array}{l}\text { Michniak et. al. J. Pharm } \\
\text { Sci. 1996, } 85 \text { (2), 150- } \\
154\end{array}$ \\
\hline SL-LACTAM-ACETIC-ESTERS/HC-4 & & 15.33 & $\begin{array}{l}\text { Michniak et. al. J. Pharm. } \\
\text { Sci. 1996, } 85 \text { (2), 150- } \\
154\end{array}$ \\
\hline SL-LACTAM-ACETIC-ESTERS/HC-5 & & 67.33 & $\begin{array}{l}\text { Michniak et. al. J. Pharm. } \\
\text { Sci. 1996, } 85 \text { (2), 150- } \\
154\end{array}$ \\
\hline SL-LACTAM-ACETIC-ESTERS/HC-6 & & 18.00 & $\begin{array}{l}\text { Michniak et. al. J. Pharm. } \\
\text { Sci. 1996, } 85 \text { (2), 150- } \\
154\end{array}$ \\
\hline SL-LACTAM-ACETIC-ESTERS/HC-7 & & 25.78 & $\begin{array}{l}\text { Michniak et. al. J. Pharm. } \\
\text { Sci. 1996, } 85 \text { (2), 150- } \\
154\end{array}$ \\
\hline SL-LACTAM-ACETIC-ESTERS/HC-8 & & 18.44 & $\begin{array}{l}\text { Michniak et. al. J. Pharm. } \\
\text { Sci. 1996, } 85 \text { (2), 150- } \\
154\end{array}$ \\
\hline SL-LACTAM-ACETIC-ESTERS/HC-9 & & 13.33 & $\begin{array}{l}\text { Michniak et. al. J. Pharm. } \\
\text { Sci. 1996, } 85 \text { (2), 150- } \\
154\end{array}$ \\
\hline SL-LACTAM-ACETIC-ESTERS/HC-10 & & 12.67 & $\begin{array}{l}\text { Michniak et. al. J. Pharm. } \\
\text { Sci. 1996, } 85 \text { (2), 150- } \\
154\end{array}$ \\
\hline SL-LACTAM-ACETIC-ESTERS/HC-11 & & 36.44 & $\begin{array}{l}\text { Michniak et. al. J. Pharm. } \\
\text { Sci. 1996, } 85 \text { (2), 150- } \\
154\end{array}$ \\
\hline SL-LACTAM-ACETIC-ESTERS/HC-12 & & 23.78 & $\begin{array}{l}\text { Michniak et. al. J. Pharm. } \\
\text { Sci. 1996, } 85 \text { (2), 150- } \\
154\end{array}$ \\
\hline SL-LACTAM-ACETIC-ESTERS/HC-13 & & 12.00 & $\begin{array}{l}\text { Michniak et. al. J. Pharm. } \\
\text { Sci. 1996, } 85 \text { (2), 150- } \\
154\end{array}$ \\
\hline SL-LACTAM-ACETIC-ESTERS/HC-14 & & 37.77 & $\begin{array}{l}\text { Michniak et. al. J. Pharm } \\
\text { Sci. 1996, } 85 \text { (2), 150- } \\
154\end{array}$ \\
\hline SL-LACTAM-ACETIC-ESTERS/HC-15 & & 1.13 & $\begin{array}{l}\text { Michniak et. al. J. Pharm } \\
\text { Sci. 1996, } 85 \text { (2), 150- } \\
154\end{array}$ \\
\hline
\end{tabular}


Table 1. (Continued)

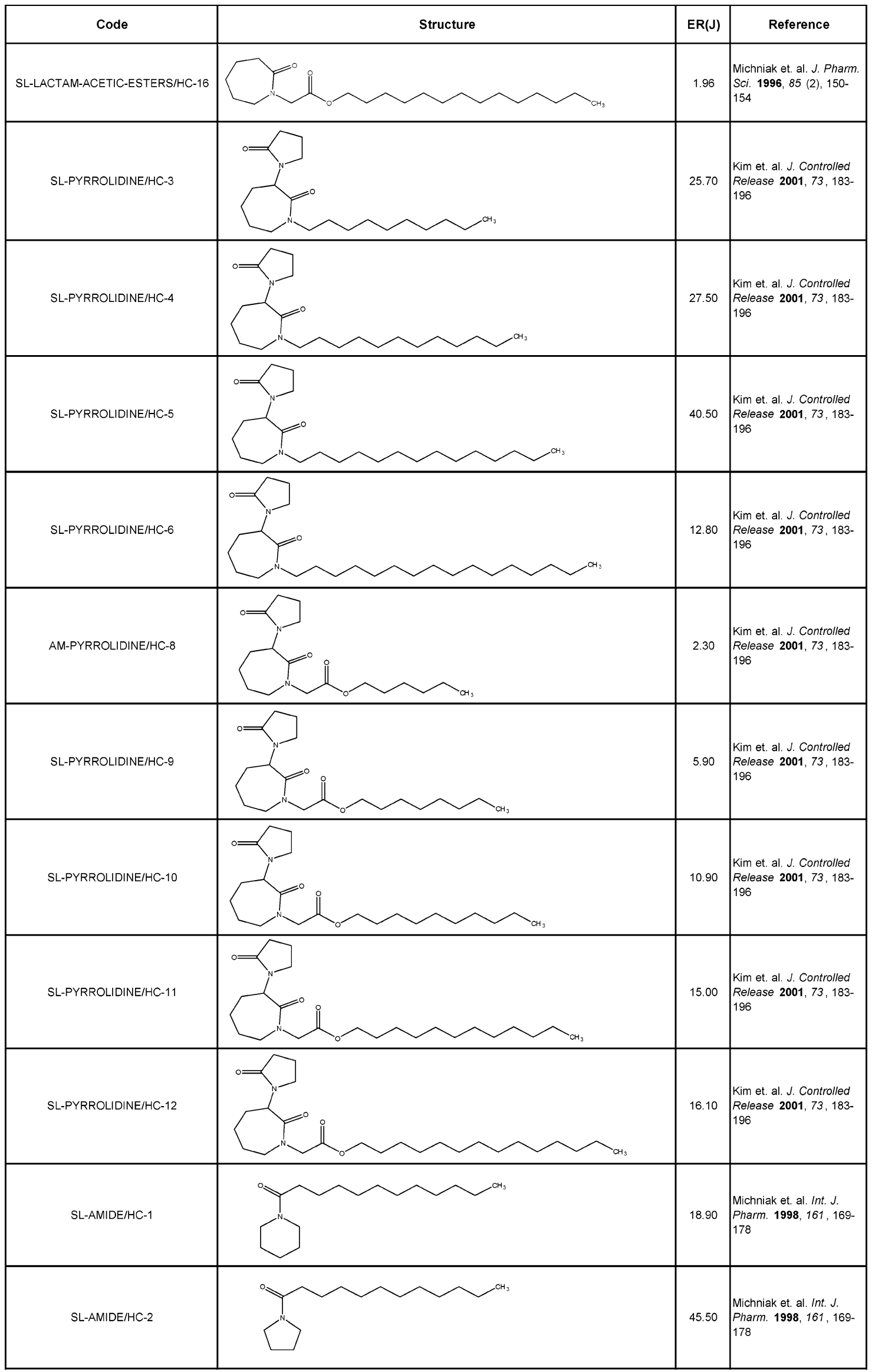


Table l. (Continued)

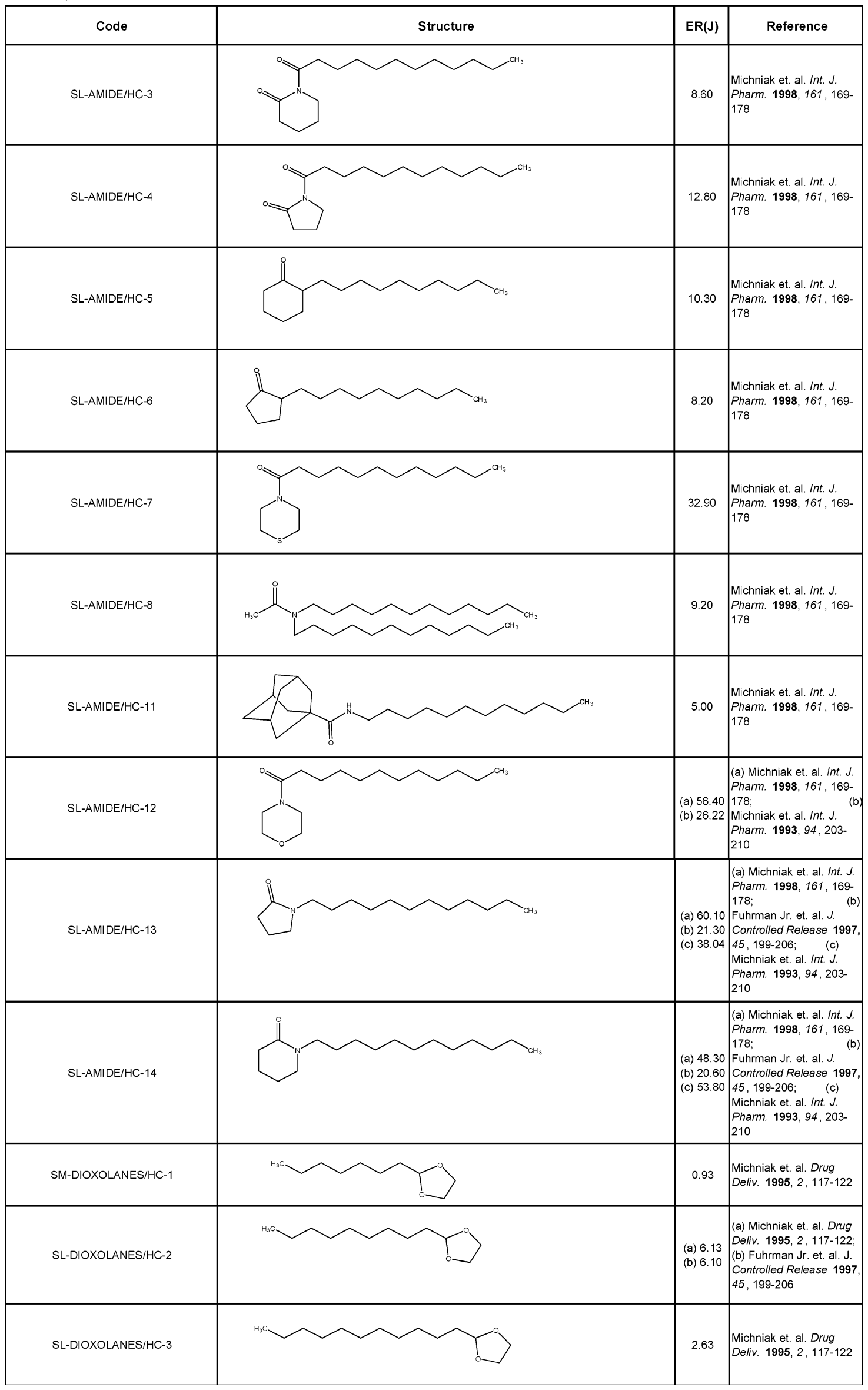


Table 1. (Continued)

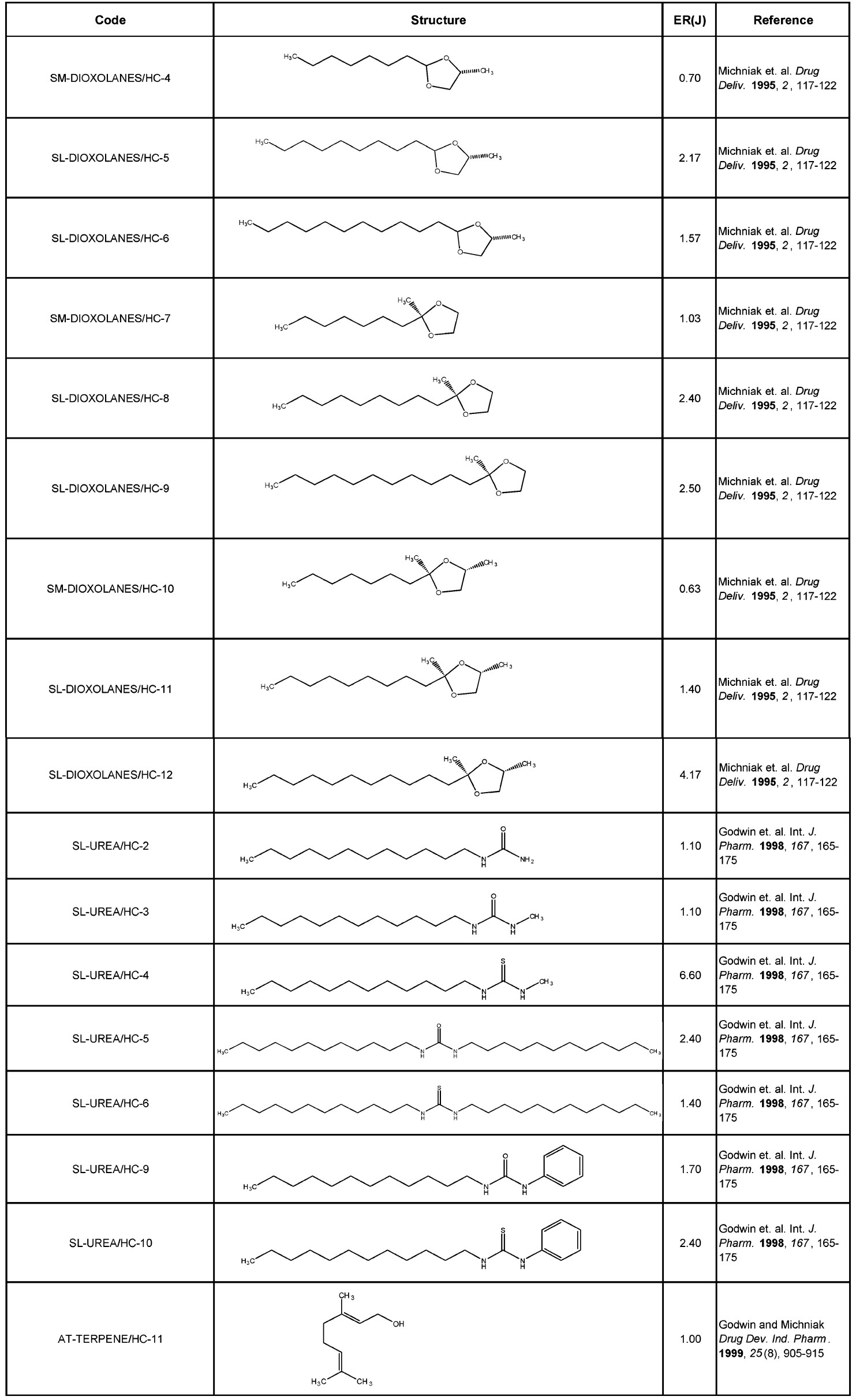


Table 1. (Continued)

\begin{tabular}{|c|c|c|c|}
\hline Code & Structure & $E R(J)$ & Reference \\
\hline SL-DIDODECYLAMINE/HC-1 & & 34.66 & $\begin{array}{l}\text { Michniak et. al. Int. J. } \\
\text { Pharm. 1995, 116, 201- } \\
\text { 209; }\end{array}$ \\
\hline SL-DODECYLAMINE/HC-2 & & 16.29 & $\begin{array}{l}\text { Michniak et. al. Int. J. } \\
\text { Pharm. 1995, 116, } 201 \\
\text { 209; }\end{array}$ \\
\hline SL-STEARYLAMINE/HC-3 & & 18.42 & $\begin{array}{l}\text { Michniak et. al. Int. J. } \\
\text { Pharm. 1995, 116, 201- } \\
\text { 209; }\end{array}$ \\
\hline
\end{tabular}

with the action of skin penetration enhancers for the nonpolar and relatively large, highly planar-shaped hydrocortisone (HC) and hydrocortisone acetate (HCA) molecules. The fourth data set involves skin penetration enhancement for the relatively small, polar fluorouracil (5FU) drug used in cancer chemotherapy. ${ }^{4}$ The two objectives reported in this paper are as follows: 1. Determine if a set of significant QSAR models can be built across a relatively large range of both skin penetration enhancement and reference penetrant chemical diversity. 2. Determine if the descriptors of the QSAR models for the penetrator enhancer systems are meaningfully different from one another, so as to suggest multiple mechanisms of skin penetration enhancement governed by the chemistry of the enhancer and/or the penetrant.

\section{METHODS}

1. Selection of Training Sets. A master data set of skin penetration enhancers was constructed from literature data. This master data set was first divided into three subsets, namely the HC subset, HCA subset, and the 5FU subset, based on the reference penetrant. Next, clustering analysis was performed on each of the three subsets based on the elements of the 2D-molecular similarity matrix of each skin penetration enhancer in each subset. The hierarchical agglomerative clustering method (HACM) of Ward, ${ }^{5}$ which employs a Euclidean distance (ED) as the similarity metric, was used to construct the "clusters" within each of the three subsets. Only the HC subset could be meaningfully clustered into two optimized clusters, while the other two subsets were found to be best represented as single clusters in terms of 2D-molecular similarity. The two $\mathrm{HC}$ clusters can be characterized as containing nonpolar, surfactant-like enhancers in one cluster and small, relatively polar enhances in the other cluster. Overall, the master data set of skin penetration enhancers partitions into four distinct penetration enhancer training sets, which are listed in Tables 1-4. References to the original skin penetration enhancer data are also given in Tables 1-4.

Training Set 1. The training set consists of 61 surfactantlike and/or nonpolar enhancers for which penetration enhancement was measured using hydrocortisone (HC) as a master reference penetrator. ${ }^{2,6-13}$ The measure of penetration enhancement, denoted by $\operatorname{ER}(J)$, is the ratio of the $\mathrm{HC}$ penetration with and without a common fixed concentration of the test enhancer. The first $\operatorname{ER}(J)$ entry for these enhancers in Tables 1-3 was used to build the QSAR models. These measurements of $\operatorname{ER}(J)$ represent the most self-consistent data because they come from the same lab or, at the very least, from one common experimental protocol. Log $\operatorname{ER}(J)$ is the actual representation for penetration enhancement used in constructing the QSAR models. This training set is given as Table 1.

The in vitro skin penetration enhancement measurement method has been described in refs 2 and 6-13. The skin permeation parameters were calculated by plotting the cumulative drug amount permeated through the skin versus the time. The slope of the linear portion of the permeation curve provided the flux value $\left(J, \mathrm{mg} \mathrm{cm}^{-2} \mathrm{~h}^{-1}\right)$ at a steady state. The $\operatorname{ER}(J)$ for flux was calculated as

$\operatorname{ER}(J)=[$ flux for skin treated with enhancer $] /$

[flux for control] (1)

where the flux for control denotes when the skin is untreated with the enhancer.

Training Set 2. This training set consists of small, relatively polar enhancers for which penetration enhancement was measured using hydrocortisone (HC) as a master reference penetrator. ${ }^{2,7,9-10,13-15}$ The measure of penetration enhancement is the same as for training set 1 . There are 44 skin penetration enhancers in this data set, which are listed as Table 2.

Training Set 3. This training set consists mainly of surfactant-like and/or nonpolar enhancers for which penetration enhancement was measured using hydrocortisone acetate (HCA) as a master reference penetrator. ${ }^{8,12-13,16-17}$ The measure of penetration enhancement is the same as for training set 1 . There are 42 skin penetration enhancers in this training set, which are given as Table 3.

Training Set 4. This training set consists of 17 terpene skin penetration enhancement compounds (polar compounds) for which penetration enhancement was measured using the chemotherapy drug $5 \mathrm{FU}$ as the master reference penetrator. ${ }^{18,19}$ The measure of penetration enhancement is the ratio of the penetration of 5FU with and without a common fixed concentration of enhancer. $\operatorname{ER}(J)$ is used to construct the QSAR models. This training set is given in Table 4.

2. Generation of QSAR Descriptors. Two classes of QSAR descriptors were generated for the skin penetration enhancers of the training sets. One class can be characterized as the "classic" intramolecular descriptors generally used in QSAR studies. The second class includes the 4D-fingerprint descriptors developed by the 4D-QSAR paradigm. ${ }^{20}$ These two classes of QSAR descriptors, in turn, permitted the development of three types of QSAR models: (a) QSAR models constructed using only the classic QSAR descriptors, 
1136 J. Chem. Inf. Model., Vol. 47, No. 3, 2007

Table 2. Training Set 2: 44 Small and Relative Polar Enhancers Using Hydrocortisone (HC) as a Master Reference Penetrator

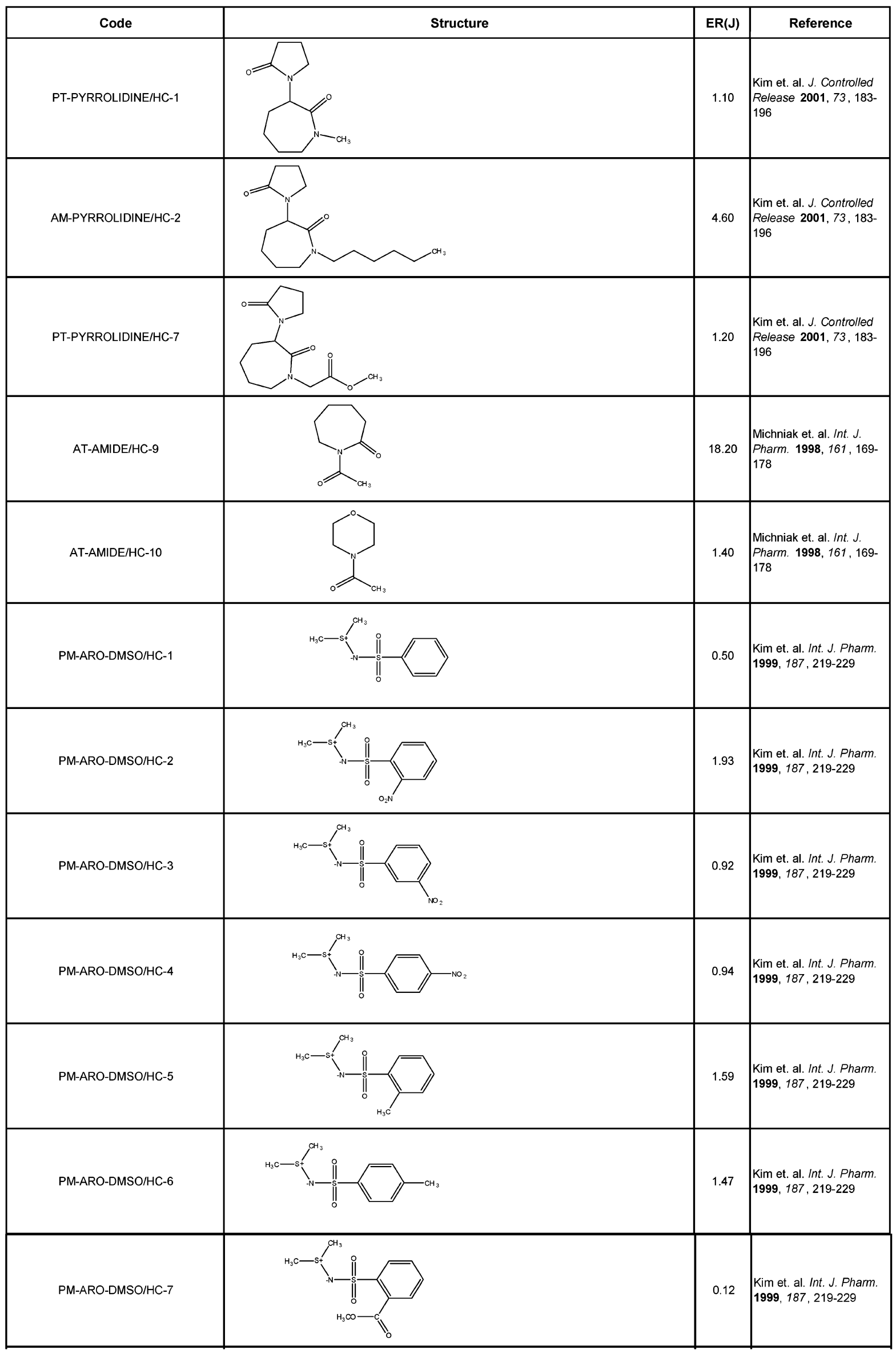


Table 2. (Continued)

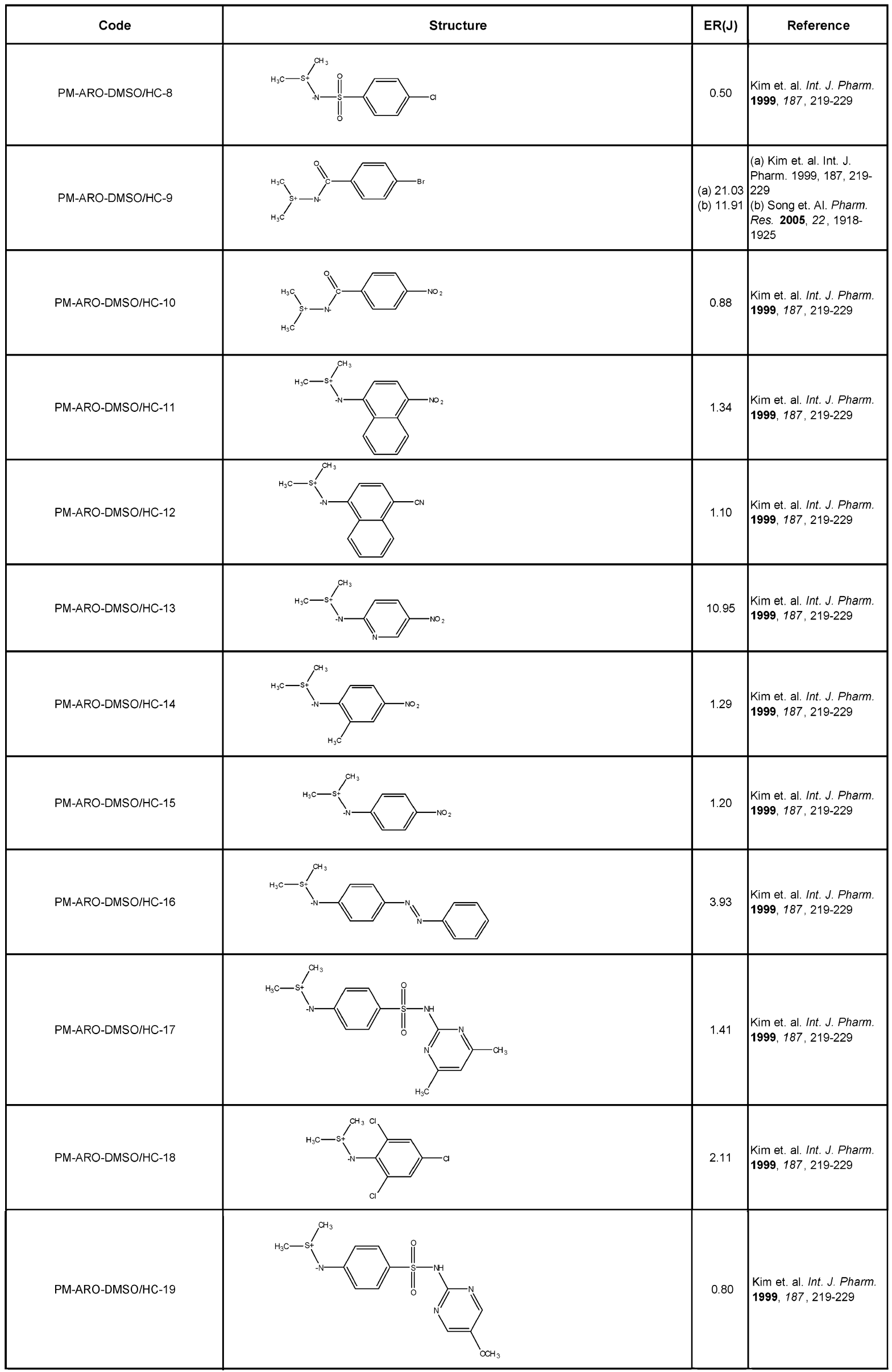


Table 2. (Continued)

\begin{tabular}{|c|c|c|c|}
\hline Code & Structure & $\operatorname{ER}(J)$ & Reference \\
\hline PT-UREA/HC-1 & & 1.20 & $\begin{array}{l}\text { Godwin et. al. Int. J. } \\
\text { Pharm. 1998, 167, 165- } \\
175\end{array}$ \\
\hline PM-UREA/HC-7 & & 2.50 & $\begin{array}{l}\text { Godwin et. al. Int. J. } \\
\text { Pharm. 1998, 167, } 165 \text { - } \\
175\end{array}$ \\
\hline PM-UREA/HC-8 & & 2.60 & $\begin{array}{l}\text { Godwin et. al. Int. J. } \\
\text { Pharm. 1998, 167, 165- } \\
175\end{array}$ \\
\hline AT-TERPENE/HC-1 & & 0.94 & $\begin{array}{l}\text { Godwin and Michniak } \\
\text { Drug Dev. Ind. Pharm. } \\
\text { 1999, 25(8), 905-915 }\end{array}$ \\
\hline AT-TERPENE/HC-2 & & 1.10 & $\begin{array}{l}\text { Godwin and Michniak } \\
\text { Drug Dev. Ind. Pharm. } \\
\text { 1999, 25(8), 905-915 }\end{array}$ \\
\hline AT-TERPENE/HC-3 & & 3.90 & $\begin{array}{l}\text { Godwin and Michniak } \\
\text { Drug Dev. Ind. Pharm. } \\
\text { 1999, 25(8), 905-915 }\end{array}$ \\
\hline AT-TERPENE/HC-4 & & 6.70 & $\begin{array}{l}\text { Godwin and Michniak } \\
\text { Drug Dev. Ind. Pharm. } \\
\text { 1999, 25(8), 905-915 }\end{array}$ \\
\hline AT-TERPENE/HC-5 & & 0.42 & $\begin{array}{l}\text { Godwin and Michniak } \\
\text { Drug Dev. Ind. Pharm. } \\
\text { 1999, 25(8), 905-915 }\end{array}$ \\
\hline AT-TERPENE/HC-6 & & 1.90 & $\begin{array}{l}\text { Godwin and Michniak } \\
\text { Drug Dev. Ind. Pharm. } \\
\text { 1999, 25(8), 905-915 }\end{array}$ \\
\hline AT-TERPENE/HC-7 & & 2.40 & $\begin{array}{l}\text { Godwin and Michniak } \\
\text { Drug Dev. Ind. Pharm. } \\
\text { 1999, 25(8), 905-915 }\end{array}$ \\
\hline AT-TERPENE/HC-8 & & 0.70 & $\begin{array}{l}\text { Godwin and Michniak } \\
\text { Drug Dev. Ind. Pharm. } \\
\text { 1999, 25(8), 905-915 }\end{array}$ \\
\hline AT-TERPENE/HC-9 & & 1.40 & $\begin{array}{l}\text { Godwin and Michniak } \\
\text { Drug Dev. Ind. Pharm. } \\
\text { 1999, 25(8), 905-915 }\end{array}$ \\
\hline
\end{tabular}


Table 2. (Continued)

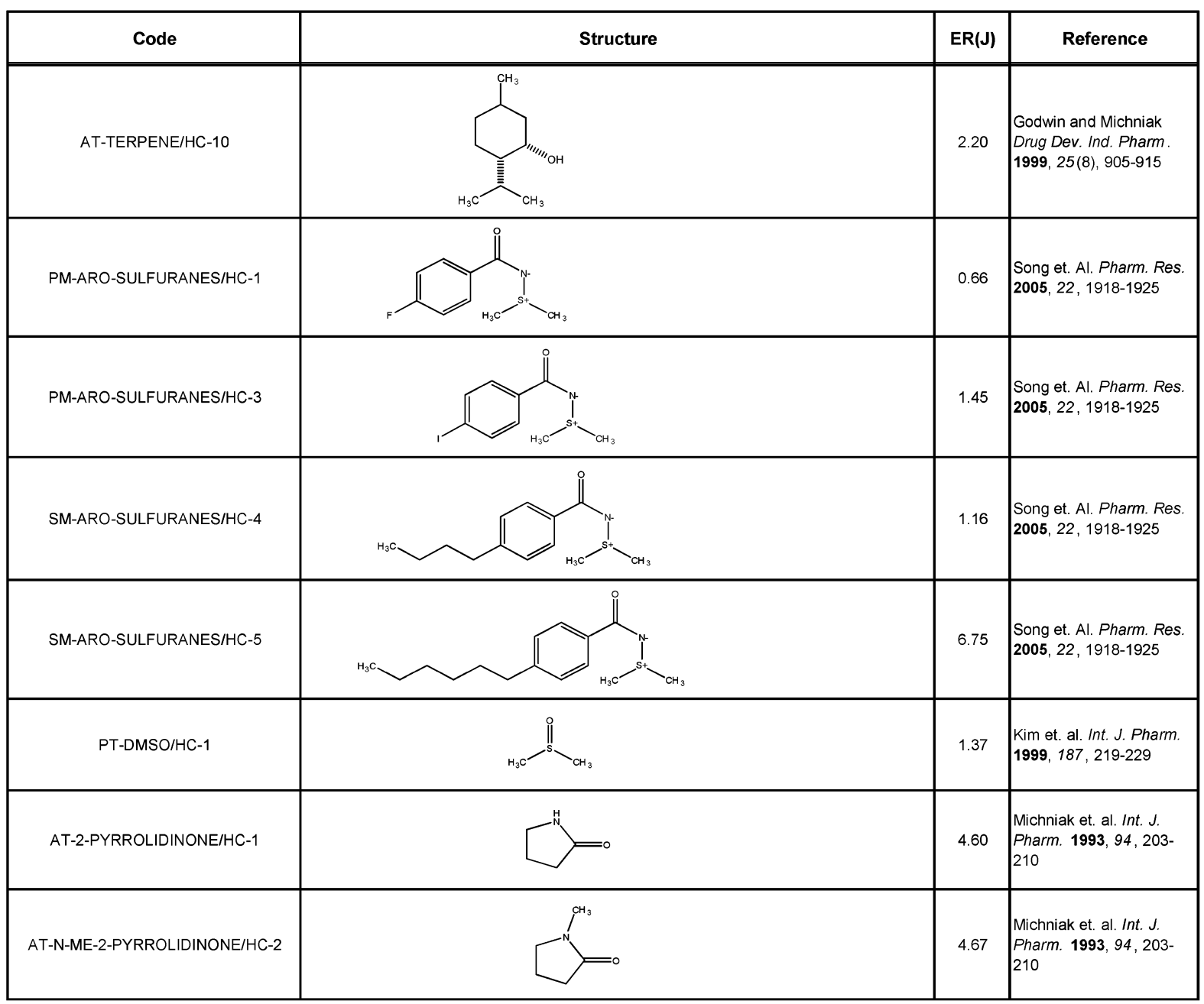

(b) QSAR models constructed using only the 4D-fingerprint descriptors, and (c) QSAR models developed using both the classic and 4D-fingerprint descriptors in the trial pool.

The set of classic QSAR descriptors used in this series of studies is given in Table 5. There may be some descriptors that other researchers consider "classic" which are not included in Table 5, but those most often used in traditional QSAR studies are listed and have been used in this work. Table 5 is divided into intramolecular and intermolecular descriptor sets as a reminder that, although these descriptors are computed from methodology based solely on the structure of an individual molecule, some are actually measures of intermolecular interactions.

The theory and methodology of the universal 4Dfingerprints have been presented in detail previously ${ }^{20,21}$ and are only summarized here. Universal 4D-fingerprints are the eigenvalues of the molecular similarity eigenvectors determined for a molecule from its absolute molecular similarity main distance-dependent matrix (MDDM). The eigenvectors capture the molecular information of a molecule regarding composition of atom types, size, shape, and conformational flexibility. The types of atoms composing a molecule are currently defined as eight interaction pharmacophore elements (IPEs), and their individual definitions are given in Table 6. A unique MDDM is constructed for each of the eight distinct and identical IPE pairs. The elements of the MDDM are derived by inductive derivation and found to be

$$
E\left(v, d_{i j}\right)=e^{\left(-v<d_{i j}>\right)}
$$

The constant $v$ in eq 2 , set to 0.25 , was selected so as to maximize the difference in the sum of eigenvalues for any two arbitrary compounds with the same number, $n$, of a particular IPE type, $m$. The term $<d_{i j}>$ is the Boltzmann conformational average distance between the atom pair $i j$ for IPE types $u$ and $v$

$$
<d_{i j}>=\sum_{k} d_{i j}(k) p(k)
$$

$p(k)$ in eq 3 is the thermodynamic probability of conformer state $k$, and it is computed from the ensemble of conformational energies determined for the molecule being studied. $d_{i j}(k)$ is the distance between atom pair $i$ and $j$ for IPE $u$ and $v$, respectively, for the $k$ th conformer state.

Diagonalization of the MDDM yields its eigenvector and constituent eigenvalues. If the members of an IPE pair are the same (i.e., $u=v$ ), MDDM is a square upper/lower triangular matrix, and it can be directly diagonalized. The resulting eigenvalues of the diagonalization are normalized, ranked in numerically descending order, and represented as an eigenvector. The $n$th normalized eigenvalue for IPE type 
Table 3. Training Set 3: 42 Surfactant-like and/or Nonpolar Enhancers Using Hydrocortisone Acetate (HCA) as a Master Reference Penetrator

\begin{tabular}{|c|c|c|c|}
\hline Code & Structure & ER(J) & Reference \\
\hline SL-ACYCLIC-AZONE-AMIDES/HCA-1 & & 13.62 & $\begin{array}{l}\text { Michniak et. al. Int. J. } \\
\text { Pharm. 1994, 110, 231- } \\
239\end{array}$ \\
\hline SL-ACYCLIC-AZONE-AMIDES/HCA-2 & $\mathrm{OCH}_{3}$ & $\begin{array}{l}\text { (a) } 20.80 \\
\text { (b) } 5.00\end{array}$ & $\begin{array}{l}\text { (a) Michniak et. al. Int. J. } \\
\text { Pharm. 1994, 110, 231- } \\
239 ; \\
\text { Fuhrman Jr. et. al. J. } \\
\text { Controlled Release 1997, } \\
\text { 45, 199-206. }\end{array}$ \\
\hline SL-ACYCLIC-AZONE-AMIDES/HCA-3 & & 20.02 & $\begin{array}{l}\text { Michniak et. al. Int. J. } \\
\text { Pharm. 1994, 110, 231- } \\
239\end{array}$ \\
\hline SL-ACYCLIC-AZONE-AMIDES/HCA-4 & & 25.78 & $\begin{array}{l}\text { Michniak et. al. Int. J. } \\
\text { Pharm. 1994, 110, 231- } \\
239\end{array}$ \\
\hline SL-ACYCLIC-AZONE-AMIDES/HCA-5 & $\mathrm{OCH}_{3}$ & $\begin{array}{l}\text { (a) } 35.22 \\
\text { (b) } 13.00\end{array}$ & $\begin{array}{l}\text { (a) Michniak et. al. Int. J. } \\
\text { Pharm. 1994, 110, 231- } \\
239 ; \\
\text { Fuhrman Jr. et. al. J. } \\
\text { Controlled Release 1997, } \\
45,199-206 .\end{array}$ \\
\hline SL-ACYCLIC-AZONE-AMIDES/HCA-6 & & 24.53 & $\begin{array}{l}\text { Michniak et. al. Int. J. } \\
\text { Pharm. 1994, 110, 231- } \\
239\end{array}$ \\
\hline SL-ACYCLIC-AZONE-AMIDES/HCA-7 & & 14.22 & $\begin{array}{l}\text { Michniak et. al. Int. J. } \\
\text { Pharm. 1994, 110, 231- } \\
239\end{array}$ \\
\hline SL-ACYCLIC-AZONE-AMIDES/HCA-8 & & 5.67 & $\begin{array}{l}\text { Michniak et. al. Int. J. } \\
\text { Pharm. 1994, 110, 231- } \\
239\end{array}$ \\
\hline SL-ACYCLIC-AZONE-AMIDES/HCA-9 & & 5.87 & $\begin{array}{l}\text { Michniak et. al. Int. J. } \\
\text { Pharm. 1994, 110, 231- } \\
239\end{array}$ \\
\hline SL-ACYCLIC-AZONE-AMIDES/HCA-10 & & 14.24 & $\begin{array}{l}\text { Michniak et. al. Int. J. } \\
\text { Pharm. 1994, 110, 231- } \\
239\end{array}$ \\
\hline SL-ACYCLIC-AZONE-AMIDES/HCA-11 & & 57.38 & $\begin{array}{l}\text { Michniak et. al. Int. J. } \\
\text { Pharm. 1994, 110, 231- } \\
239\end{array}$ \\
\hline SL-ACYCLIC-AZONE-AMIDES/HCA-12 & & 29.38 & $\begin{array}{l}\text { Michniak et. al. Int. J. } \\
\text { Pharm. 1994, 110, 231- } \\
239\end{array}$ \\
\hline SL-ACYCLIC-AZONE-AMIDES/HCA-13 & & 6.51 & $\begin{array}{l}\text { Michniak et. al. Int. J. } \\
\text { Pharm. 1994, 110, 231- } \\
239\end{array}$ \\
\hline
\end{tabular}


Table 3. (Continued)

\begin{tabular}{|c|c|c|c|}
\hline Code & Structure & $E R(J)$ & Reference \\
\hline SL-ACYCLIC-AZONE-AMIDES/HCA-14 & 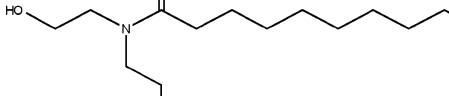 & 10.02 & $\begin{array}{l}\text { Michniak et. al. Int. J. } \\
\text { Pharm. 1994, 110, 231- } \\
239\end{array}$ \\
\hline SL-AZONE-AMINES/HCA-1 & & 11.80 & $\begin{array}{l}\text { Michniak et. al. Int. J. } \\
\text { Pharm. 1995, 116, 201- } \\
\text { 209; }\end{array}$ \\
\hline SL-AZONE-AMINES/HCA-2 & & 16.00 & $\begin{array}{l}\text { Michniak et. al. Int. J. } \\
\text { Pharm. 1995, 116, 201- } \\
\text { 209; }\end{array}$ \\
\hline SL-AZONE-AMINES/HCA-3 & & 13.18 & $\begin{array}{l}\text { Michniak et. al. Int. J. } \\
\text { Pharm. 1995, 116, } 201 \text { - } \\
\text { 209; }\end{array}$ \\
\hline SL-AZONE-AMINES/HCA-4 & & 9.16 & $\begin{array}{l}\text { Michniak et. al. Int. J. } \\
\text { Pharm. 1995, 116, 201- } \\
\text { 209; }\end{array}$ \\
\hline SL-AZONE-AMINES/HCA-5 & & 30.89 & $\begin{array}{l}\text { Michniak et. al. Int. J. } \\
\text { Pharm. 1995, 116, 201- } \\
\text { 209; }\end{array}$ \\
\hline SL-AZONE-AMINES/HCA-6 & & 8.04 & $\begin{array}{l}\text { Michniak et. al. Int. J. } \\
\text { Pharm. 1995, 116, 201- } \\
\text { 209; }\end{array}$ \\
\hline SL-AZONE-AMINES/HCA-7 & & 17.80 & $\begin{array}{l}\text { Michniak et. al. Int. J. } \\
\text { Pharm. 1995, 116, 201- } \\
\text { 209; }\end{array}$ \\
\hline SL-AZONE-AMINES/HCA-8 & & 9.87 & $\begin{array}{l}\text { Michniak et. al. Int. J. } \\
\text { Pharm. 1995, 116, 201- } \\
\text { 209; }\end{array}$ \\
\hline SL-AZONE-AMINES/HCA-9 & & 15.33 & $\begin{array}{l}\text { Michniak et. al. Int. J. } \\
\text { Pharm. 1995, 116, 201- } \\
\text { 209; }\end{array}$ \\
\hline SL-AZONE-AMINES/HCA-10 & & 25.56 & $\begin{array}{l}\text { Michniak et. al. Int. J. } \\
\text { Pharm. 1995, 116, 201- } \\
\text { 209; }\end{array}$ \\
\hline SL-AZONE-AMINES/HCA-11 & & $\begin{array}{l}\text { (a) } 16.11 \\
\text { (b) } 7.00\end{array}$ & $\begin{array}{l}\text { (a) Michniak et. al. Int. J. } \\
\text { Pharm. 1995, 116, 201- } \\
\text { 209; } \\
\text { (b) Fuhrman Jr. et. al. J. } \\
\text { Controlled Release 1997, } \\
45,199-206 . \\
\end{array}$ \\
\hline SL-AZONE-AMINES/HCA-12 & & 13.84 & $\begin{array}{l}\text { Michniak et. al. Int. J. } \\
\text { Pharm. 1995, 116, 201- } \\
\text { 209; }\end{array}$ \\
\hline SL-AZONE-THIO-AMIDE/HCA-3 & & 13.71 & $\begin{array}{l}\text { Michniak et. al. Int. J. } \\
\text { Pharm. 1993, 94, 203- } \\
210\end{array}$ \\
\hline SL-AZONE-THIO-AMIDE/HCA-4 & & 8.56 & $\begin{array}{l}\text { Michniak et. al. Int. J. } \\
\text { Pharm. 1993, 94, 203- } \\
210\end{array}$ \\
\hline SL-AZONE-THIO-AMIDE/HCA-5 & & 4.33 & $\begin{array}{l}\text { Michniak et. al. Int. J. } \\
\text { Pharm. 1993, 94, 203- } \\
210\end{array}$ \\
\hline
\end{tabular}


Table 3. (Continued)

\begin{tabular}{|c|c|c|c|}
\hline Code & Structure & $\operatorname{ER}(J)$ & Reference \\
\hline SL-AZONE-THIO-AMIDE/HCA-7 & & 34.02 & $\begin{array}{l}\text { Michniak et. al. Int. J. } \\
\text { Pharm. 1993, 94, 203- } \\
210\end{array}$ \\
\hline SL-AZONE-THIO-AMIDE/HCA-8 & & 2.09 & $\begin{array}{l}\text { Michniak et. al. Int. J. } \\
\text { Pharm. 1993, 94, 203- } \\
210\end{array}$ \\
\hline SL-AZONE-THIO-AMIDE/HCA-9 & & 7.56 & $\begin{array}{l}\text { Michniak et. al. Int. J. } \\
\text { Pharm. 1993, 94, 203- } \\
210\end{array}$ \\
\hline SL-AZONE/HCA-3 & & 7.09 & $\begin{array}{l}\text { Michniak et. al. Int. J. } \\
\text { Pharm. 1993, 91, 85-93 }\end{array}$ \\
\hline SL-AZONE/HCA-4 & & 5.20 & $\begin{array}{l}\text { Michniak et. al. Int. J. } \\
\text { Pharm. 1993, 91, 85-93 }\end{array}$ \\
\hline SL-AZONE/HCA-5 & & 44.96 & $\begin{array}{l}\text { Michniak et. al. Int. J. } \\
\text { Pharm. 1993, 91, 85-93 }\end{array}$ \\
\hline SL-AZONE/HCA-6 & & 12.67 & $\begin{array}{l}\text { Michniak et. al. Int. J. } \\
\text { Pharm. 1993, 91, 85-93 }\end{array}$ \\
\hline SL-AZONE/HCA-7 & & 10.20 & $\begin{array}{l}\text { Michniak et. al. Int. J. } \\
\text { Pharm. 1993, 91, 85-93 }\end{array}$ \\
\hline SL-AZONE/HCA-8 & & 14.07 & \begin{tabular}{|l} 
Michniak et. al. Int. J. \\
Pharm. 1993, 91, 85-93
\end{tabular} \\
\hline SL-AZONE/HCA-9 & & 18.80 & $\begin{array}{l}\text { Michniak et. al. Int. J. } \\
\text { Pharm. 1993, 91, 85-93 }\end{array}$ \\
\hline SL-AZONE/HCA-10 & & 12.24 & $\begin{array}{l}\text { Michniak et. al. Int. J. } \\
\text { Pharm. 1993, 91, 85-93 }\end{array}$ \\
\hline SL-AZONE/HCA-11 & & 14.38 & $\begin{array}{l}\text { Michniak et. al. Int. J. } \\
\text { Pharm. 1993, 91, 85-93 }\end{array}$ \\
\hline SL-AZONE/HCA-12 & & 20.00 & $\begin{array}{l}\text { Michniak et. al. Int. J. } \\
\text { Pharm. 1993, 91, 85-93 }\end{array}$ \\
\hline
\end{tabular}

m of a compound $\alpha, \epsilon_{\mathrm{mn}}(\alpha)$, is obtained by scaling the nonnormalized eigenvalue $\epsilon_{\mathrm{mn}}{ }^{\prime}(\alpha)$ relative to the rank of its MDDM
If the members of an IPE pair are not the same (i.e., $u \neq v$ ), the number of $u$ and $v$ IPE elements can be different $\left(n_{u} \neq n_{v}\right)$. In this case, MDDM will be rectangular, 
Table 4. Training Set 4: The Terpene Training Set Using 5-FU as the Polar Reference Transport Molecule

\begin{tabular}{|c|c|c|c|c|}
\hline Compound\# & Compound Name & Structure & $\operatorname{ER}(J)$ & Reference \\
\hline 1 & d-limonene & & 2.1 & $\begin{array}{l}\text { William, A; Bary B. Pham. } \\
\text { Res. 1991, 8, 17-24 }\end{array}$ \\
\hline 2 & alpha-pinene & & 1.2 & $\begin{array}{l}\text { William, A; Bary B. Pham. } \\
\text { Res. 1991, 8, 17-24 }\end{array}$ \\
\hline 3 & 3-carene & & 3.2 & $\begin{array}{l}\text { William, A; Bary B. Pham. } \\
\text { Res. 1991, 8, 17-24 }\end{array}$ \\
\hline 4 & alpha-terpinene & & 9.4 & $\begin{array}{l}\text { William, A; Bary B. Pham. } \\
\text { Res. 1991, 8, 17-24 }\end{array}$ \\
\hline 5 & terpinen-4-d & & 10.4 & $\begin{array}{l}\text { William, A; Bary B. Pham. } \\
\text { Res. 1991, 8, 17-24 }\end{array}$ \\
\hline 6 & carveol & & 20 & $\begin{array}{l}\text { William, A; Bary B. Pham. } \\
\text { Res. 1991, 8, 17-24 }\end{array}$ \\
\hline 7 & carvone & & 12.2 & $\begin{array}{l}\text { William, A; Bary B. Pham. } \\
\text { Res. 1991, 8, 17-24 }\end{array}$ \\
\hline 8 & pulegone & & 21.2 & $\begin{array}{l}\text { William, A; Barry B. Pharm. } \\
\text { Res. 1991, } 8,17-24\end{array}$ \\
\hline 9 & piperitone & & 27.7 & $\begin{array}{l}\text { William, A; Barry B. Pharm. } \\
\text { Res. 1991, 8, 17-24 }\end{array}$ \\
\hline
\end{tabular}


Table 4. (Continued)

\begin{tabular}{|c|c|c|c|c|}
\hline Compound \# & Compound Name & Structure & $\operatorname{ER}(J)$ & Reference \\
\hline 10 & menthone & & 37.9 & $\begin{array}{l}\text { William, A; Barry B. Pharm. } \\
\text { Res. 1991, 8, 17-24 }\end{array}$ \\
\hline 11 & cyclohexene oxide & & 2.4 & $\begin{array}{l}\text { William, A; Barry B. Pharm. } \\
\text { Res. 1991, 8, 17-24 }\end{array}$ \\
\hline 12 & limonene oxide & & 11 & $\begin{array}{l}\text { William, A; Barry B. Pharm. } \\
\text { Res. 1991, 8, 17-24 }\end{array}$ \\
\hline 13 & alpha-pinene-oxide & & 13.7 & $\begin{array}{l}\text { William, A; Barry B. Pharm. } \\
\text { Res. 1991, 8, 17-24 }\end{array}$ \\
\hline 14 & cyclopentene-oxide & & 30.9 & $\begin{array}{l}\text { William, A; Barry B. Pharm. } \\
\text { Res. 1991, 8, 17-24 }\end{array}$ \\
\hline 15 & ascaridole & & 82.5 & $\begin{array}{l}\text { William, A; Barry B. Pharm. } \\
\text { Res. 1991, 8, 17-24 }\end{array}$ \\
\hline 16 & 7-oxabicyclo $[2,2,1]$-heptane & & 91.7 & $\begin{array}{l}\text { William, A; Barry B. Pharm. } \\
\text { Res. 1991, 8, 17-24 }\end{array}$ \\
\hline 17 & 1,8 -cineole & & 94.5 & $\begin{array}{l}\text { William, A; Barry B. Pharm. } \\
\text { Res. 1991, 8, 17-24 }\end{array}$ \\
\hline
\end{tabular}

but the following two square MDDMs can still be constructed:

$$
\begin{aligned}
& \operatorname{MDDM}(u, u)=\operatorname{MDDM}\left(n_{u} n_{v}\right) * \operatorname{MDDM}\left(n_{u}, n_{v}\right)^{T} \\
& \operatorname{MDDM}(v, v)=\operatorname{MDDM}\left(n_{v} n_{u}\right) * \operatorname{MDDM}\left(n_{v}, n_{u}\right)^{T}
\end{aligned}
$$

$\operatorname{MDDM}(u, u)$ and $\operatorname{MDDM}(v, v)$ have the same set of eigenvalues because they have the same rank and trace. As a result, for each IPE pair $u \neq v$

$$
\epsilon(\alpha)_{u, v}=\left\{[\epsilon(\alpha)]_{\operatorname{MDDM}(u, u)}\right\}^{1 / 2}
$$

Each IPE pair corresponds to one MDDM from which one molecular similarity eigenvector can be formed. Since there are 36 distinct combinations of the currently defined eight IPE types, 36 eigenvectors can be obtained for each molecule $\alpha$. Dissimilarity between molecules $\alpha$ and $\beta$ is given by

$$
D_{\alpha \beta}=\sum_{i}\left|\epsilon(\alpha)_{i}-\epsilon(\beta)_{i}\right|
$$

where $i$ refers to the $i$ th eigenvalue in the corresponding eigenvector of a specific IPE pair. Molecular similarity is then defined as

$$
S_{\alpha \beta}=\left(1-D_{\alpha \beta}\right)(1-\varphi)
$$

where $\varphi=|\operatorname{rank}(\alpha)-\operatorname{rank}(\beta)| /(\operatorname{rank}(\alpha)+\operatorname{rank}(\beta))$. Since 
Table 5. Classic Set of QSAR Descriptors

\begin{tabular}{ll}
\hline & \multicolumn{1}{c}{ Intramolecular } \\
HOMO & highest occupied molecular orbital energy \\
LUMO & lowest unoccupied molecular orbital energy \\
Dp & dipole moment \\
Vm & molecular volume \\
$\mathrm{SA}$ & molecular surface area \\
$\mathrm{Ds}$ & density \\
$\mathrm{MW}$ & molecular weight \\
$\mathrm{MR}$ & molecular refractivity \\
$N(\mathrm{hba})$ & number of hydrogen bond acceptors \\
$N(\mathrm{hbd})$ & number of hydrogen bond donors \\
$N(\mathrm{~B})$ & number of rotatable bonds \\
$\mathrm{JSSA}(\mathrm{X})$ & Jurs-Stanton surface area descriptors \\
$\mathrm{Chi}-\mathrm{N}, \mathrm{Kappa}-\mathrm{M}$ & Kier and Hall topological descriptors \\
$\mathrm{Rg}$ & radius of gyration \\
$\mathrm{PM}$ & principle moment of inertia \\
$\mathrm{PSA}$ & polar surface area \\
$\mathrm{Se}$ & conformational entropy \\
$Q(\mathrm{I})$ & partial atomic charge densities \\
& $\quad$ Intermolecular \\
$F\left(\mathrm{H}_{2} \mathrm{O}\right)$ & aqueous solvation free energy \\
$F($ oct $)$ & 1-octanol solvation free energy \\
LogP & 1-octanol/water partition coefficient \\
$E_{\mathrm{coh}}$ & cohesive packing energy \\
$T_{\mathrm{M}}$ & hypothetical crystal-melt transition temperature \\
$T_{\mathrm{G}}$ & hypothetical glass transition temperature \\
&
\end{tabular}

Table 6. Interaction Pharmacophore Elements, IPEs, Currently Used in the 4D-QSAR Paradigm

\begin{tabular}{ccl}
\hline IPE code & IPE abbreviation & \multicolumn{1}{c}{ IPE description } \\
\hline 0 & any & all atoms in the molecule \\
1 & np & nonpolar atoms \\
2 & $\mathrm{p}+$ & polar $(+)$ atoms \\
3 & $\mathrm{p}-$ & polar $(-)$ atoms \\
4 & hba & hydrogen bond acceptor atoms \\
5 & hbd & hydrogen bond donor atoms \\
6 & aro & aromatic atoms \\
7 & hs & non-hydrogen atoms
\end{tabular}

${ }^{a}$ Sometimes a carbon or sulfur will end of having a relatively large negative partial atomic charge $[<-0.150]$ owing to the bonding topology in which it is involved. In these cases the 4D-QSAR paradigm considers the carbon or sulfur to be a polar negative IPE as opposed to a nonpolar IPE.

$$
\epsilon_{\mathrm{mn}}(\alpha)=\frac{\epsilon_{\mathrm{mn}}{ }^{\prime}(\alpha)}{\operatorname{rank}(\alpha)_{\mathrm{m}}}
$$

the rank of a MDDM matrix is the number of atoms of the specific IPE type present, $\varphi$ serves to reincorporate molecular size information into $S_{\mathrm{ab}}$. The normalized eigenvalues limit the range of both $D_{\alpha \beta}$ and $S_{\alpha \beta}$ between 0 and 1 . When a $D_{\alpha \beta}$ value is closer to 1 , it means a higher degree of dissimilarity, whereas an $S_{\alpha \beta}$ value closer to 1 means higher molecular similarity.

The universal 4D-fingerprint descriptor set for molecule $\alpha$ is composed of all eigenvalues of all eigenvectors derived from all of the MDDM for $\alpha$. Operationally, a threshold cutoff value of 0.002 is applied to disregard normalized eigenvalues less than the threshold.

Construction of the trial descriptor matrix for all training set compounds is based on maximizing its information content. For each compound in the training set, the number of significant eigenvalues in the eigenvector for a particular IPE pair $(u, v)$ is first computed. Then, the maximum number of significant eigenvalues, $n_{\max }(u, v)$, across the training set
Table 7. Results of the Random Scramblings Experiments and the Findings from the Test Set Predictions Studies for the Original Four Training Sets ${ }^{a}$

\begin{tabular}{cll}
\hline \multicolumn{1}{c}{ data set } & \multicolumn{1}{c}{$\left\langle r^{2}\right\rangle$} & $\left\langle q^{2}\right\rangle$ \\
\hline original training set 1: $N=61$ & 0.29 - scrambled & \\
original training set 2: $N=44$ & 0.36 - scrambled & \\
original training set 3: $N=42$ & 0.23 - scrambled & \\
original training set 4: $N=17$ & 0.37 - scrambled & \\
test training set 1: $N=52$ & 0.788 - eq 11/-15\% & 0.711 \\
test training set 2: $N=37$ & 0.802 - eq 11/-15\% & 0.735 \\
test training set 3: $N=36$ & 0.782 - eq 11/-15\% & 0.744 \\
test training set 4: $N=14$ & 0.856 - eq 11/-15\% & 0.772 \\
test set 1: $N=9$ & 0.68 & \\
test set 2: $N=7$ & 0.70 & \\
test set 3: $N=6$ & 0.67 & \\
test set 4: $N=3$ & 0.78 &
\end{tabular}

${ }^{a}$ The "test training sets" are those data sets constructed from randomly extracting $15 \%$ of the compounds in the original training set and using the resultant data set to build a QSAR model. The "test sets" are the $15 \%$ of the compounds extracted from the original training set and whose activities are predicted using QSAR models built from the remaining $85 \%$ of the compounds in the original training set. $N$ in each case is the number of compounds in the data set. The $\left.<r^{2}\right\rangle$ are for the 4000 random scrambling level as described in the test.

is determined. Finally, all molecules in the training set are assigned $n_{\max }(u, v)$ eigenvalues from their corresponding eigenvectors for the IPE pair $(u, v)$. Eigenvectors containing less than $n_{\max }(u, v)$ significant eigenvalues have these "missing" eigenvalues set to zero. For instance, if $n_{\max }(3,5)$ is 10 , and the eigenvector for IPE pair $(u, v)$ of compound $\alpha$ has only eight significant eigenvalues, the ninth and tenth eigenvalues for IPE pair $(u, v)$ of $\alpha$ are set to zero.

The total number of universal descriptors, $n_{\text {total }}$, for each compound in the training set will be the sum of the $n_{\max }-$ $(u, v)$ values for the 36 eigenvectors. Each such universal descriptor $\epsilon_{i}(u, v)$ represents the $i$ th eigenvalue in the eigenvector for the IPE pair $(u, v)$.

3. Construction of the QSAR Models. The skin penetration-enhancer models were built and optimized using multidimensional linear regression fitting and genetic function approximation (GFA), which is a multidimensional optimization method based on the genetic algorithm paradigm..$^{22,23}$ Both linear and quadratic representations for each of the descriptor values were included in the trial descriptor pool, and QSAR models were built as a function of the number of descriptor terms in a given model. Statistical significance in the optimization of a QSAR model was judged jointly by the correlation coefficient of fit, $r^{2}$, and the leave-one-out cross validation correlation coefficient, $q^{2}$. In addition, GFA uses the Friedman's lack of fit (LOF) measure to resist overfitting, which is a problem often encountered in constructing statistical models. ${ }^{24}$

4. Exploration and Validation of the QSAR Models. Each of the four original training sets was repeatedly subdivided, on a random selection basis, into a subtraining set and a test set. Each test set was restricted to $15 \%$ of the compounds in the original training set. Ten such subtraining sets and test sets were constructed for each of the four original training sets. QSAR models were constructed in identical fashion as those in the original training set for each corresponding subtraining set. Each resulting QSAR model was then used to predict the corresponding test set com- 
pounds. The average $r^{2}$ and $q^{2}$ of the subtraining set QSAR models and the average $r^{2}$ of prediction for the test set compounds are reported in Table 7.

The dependent variables, $\operatorname{ER}(J)$, of each of the four original training sets were randomly scrambled in increments of 1000 beginning with 1000 random scramblings. In each instance a QSAR model was constructed to test for chance correlations. By the 4000 random scrambling level, there was no meaningful change in the average $r^{2}$ value, or its mean deviation, across the QSAR models of the random scramblings as compared to the corresponding measures from the models of 2000 and 3000 random scrambling levels for all four training sets. The average $r^{2}$ values, from the 4000 random scrambling experiments, across each of the four training sets range from 0.23 to 0.37 . Thus, 4000 random scramblings are considered sufficient to establish that the models generated in this study are not due to chance. The results, in terms of the average $r^{2}$ value across the QSAR models of the random scramblings, at the 4000 randomscrambling level, are also given in Table 7.

\section{RESULTS}

An attempt to recombine training sets 1 and 2, both using $\mathrm{HC}$ as the reference penetrant to build a single composite QSAR model, was not possible; no statistically significant QSAR could be constructed for the combination of the two training sets, thus suggesting that nonpolar, surfactant-like penetrants [training set 1] may act by a different mechanism of $\mathrm{HC}$ transport enhancement than is employed by the relatively small, polar enhancers [training set 2].

Training sets 1 and 3 were also combined to see if a single QSAR model could be built. The chemical structures of the enhancers in these two training sets are generally similar but do differ in that $\mathrm{HC}$ is the reference penetrant in training set 1, while HCA is the reference penetrant for training set 3 . Once again, no significant single QSAR model could be built by combining training sets 1 and 3 . This finding, again, may be indicative of a somewhat different mechanism, or transport path, of HC versus HCA through the stratum corneum.

The optimized QSAR models for training set 1, which consists of surfactant-like and/or nonpolar enhancers with $\mathrm{HC}$ as the master reference penetrator, are as follows: (a) models constructed using only the classic QSAR descriptors, (b) models constructed using only the 4D-fingerprint descriptors, and (c) models developed using both the classic and 4D-fingerprint descriptors in the trial descriptor pool.

The definitions of the classic QSAR descriptors for all of the optimum QSAR models are listed in Table 5, and the IPE [atom types] of the 4D-fingerprint descriptors are defined in Table 6.

The trial descriptor pools used to build the QSAR models of training set 1 consisted of 79 classic QSAR descriptors and 441 4D-fingerprint descriptors.

\section{A. Classic QSAR Descriptors.}

$$
\begin{array}{r}
\log \operatorname{ER}(J)=2.013+0.039 *(\mathrm{Clog} \mathrm{P}-8.329)^{2}- \\
0.0001 *(\mathrm{HOMO}+242.46)^{2}+0.004 *(\mathrm{Kappa} 5- \\
10)^{2}-0.008 *(\mathrm{FH} 20-2.48)^{2}-0.019 *(\mathrm{Kappa} 4- \\
15.71)^{2}-3.259 * \text { Dipole }(10)
\end{array}
$$

$$
N=61, r^{2}=0.732, q^{2}=0.661
$$

\section{B. 4D-Fingerprint QSAR Descriptors.}

$$
\begin{gathered}
\log \operatorname{ER}(J)=2.823-2.415 * \mathrm{e} 2(\mathrm{p}-\text {, any })- \\
96.37 * \mathrm{e} 7(\text { any,any })-137.8 * \mathrm{e} 12(\mathrm{np}, \mathrm{np})+ \\
74.66 * \mathrm{e} 18(\mathrm{hs}, \mathrm{hs})+216.9 * \mathrm{e} 16(\text { any }, \text { any }) \\
N=61, r^{2}=0.743, q^{2}=0.673
\end{gathered}
$$

\section{Classic QSAR and 4D-Fingerprint Descriptors.}

$$
\begin{gathered}
\log \operatorname{ER}(J)=1.143+48.27 * \mathrm{e} 18(\mathrm{hs}, \mathrm{hs})+ \\
34.75 * \mathrm{e} 4(\mathrm{hs}, \mathrm{hs})+0.076 * \mathrm{FH} 2 \mathrm{O}-0.155 * \mathrm{PSA}- \\
384.7 * \mathrm{e} 22(\text { any,any })+1.458 * \mathrm{e} 1(\mathrm{p}+, \mathrm{hs})(12) \\
N=61, r^{2}=0.767, q^{2}=0.724
\end{gathered}
$$

Regardless of which descriptors are used to build the QSAR models, the optimal number of terms in a model, as determined from GFA, is always six. The 4D-fingerprints by themselves lead to a marginally better model than that arising solely from classic QSAR descriptors. However, the all-4D-fingerprint QSAR model, eq 11, has only linear descriptor terms, while five of the six terms in eq 10 (the classic descriptor QSAR model) are quadratic. This suggests that the 4D-fingerprints better fit the $\log \operatorname{ER}(J)$ measures. Equation 12, which is the QSAR model built from combining the classic and 4D-fingerprint descriptors, proves marginally better in $r^{2}$ than eqs 10 and 11 but is more superior in $q^{2}$ than the other two QSAR models. Moreover, all terms in eq 12 are linear, including those of the classic descriptors, and only one descriptor from each of eqs 10 and 11 is found in eq 12.

The optimized QSAR models derived from the second training set are given below in the same format used to report the QSAR models of training set 1. The trial descriptor pools used to build the models consisted of 76 classic QSAR descriptors and 357 4D-fingerprint descriptors.

Classic QSAR Descriptors. No significant QSAR model could be constructed using only classic QSAR descriptors.

4D-Fingerprint QSAR Descriptors.

$\log \operatorname{ER}(J)=0.316-2378$. * (e4(any,any) -0.056$)^{2}-$ $59.19 *(\mathrm{e} 3(\text { any,np }))^{2}-270.1 *(\mathrm{e} 3(\mathrm{np}, \mathrm{np})-0.079)^{2}-$ $0.238 *(\mathrm{e} 1(\mathrm{np}, \mathrm{hba})-1.00)^{2}+4698 . *(\mathrm{e} 16($ any,np $)-$

$$
\begin{aligned}
& 0.014)^{2}+7617 *(\text { e } 37(\text { any }, \text { any }))^{2}(13) \\
& N=44, r^{2}=0.772, q^{2}=0.710
\end{aligned}
$$

Classic QSAR and 4D-Fingerprint Descriptors - Same as Eq 13.

$$
\begin{gathered}
\log \operatorname{ER}(J)=0.316-2378 . *(\mathrm{e} 4(\text { any,any })-0.056)^{2}- \\
59.19 *(\mathrm{e} 3(\text { any,np }))^{2}-270.1 *(\mathrm{e} 3(\mathrm{np}, \mathrm{np})-0.079)^{2}- \\
0.238 *(\mathrm{e} 1(\mathrm{np}, \mathrm{hba})-1.00)^{2}+4698 *(\mathrm{e} 16(\text { any, np })- \\
0.014)^{2}+7617 . *(\mathrm{e} 37(\text { any,any }))^{2}(14) \\
N=44, r^{2}=0.772, q^{2}=0.710
\end{gathered}
$$

In this part of the study, it was found that no significant QSAR model could be built using only classic QSAR 
descriptors, and when the classic and 4D-fingerprint descriptor pools were combined, the resulting model was the same as that built using only 4D-fingerprints. Once again the optimal size of the QSAR model is six terms, all of which are quadratic. Even though model construction seems more demanding for this training set than for training set 1 , the $r^{2}$ and $q^{2}$ of eq 13, respectively, are about the same as those in eq 12 .

The optimal QSAR models for training set 3 are based on descriptor pools of 77 classic descriptors and 413 4Dfingerprint descriptors. They are as follows:

\section{Classic QSAR Descriptors.}

$$
\begin{gathered}
\log \operatorname{ER}(J)=1.848-0.002 * T_{\mathrm{G}}-0.0002 *(\mathrm{PSA}- \\
21.7)^{2}-0.0001 *(\mathrm{LUMO}-111.2)^{2}-0.00001 * \\
\left(T_{\mathrm{M}}-444.7\right)^{2}-2.519 *(\mathrm{Chi} 11-0.27)^{2}+ \\
0.781 * \text { Dipole }+0.011 *(\text { Kappa } 5-11.23)^{2} \\
N=42, r^{2}=0.738, q^{2}=0.621
\end{gathered}
$$

\section{D-Fingerprint QSAR Descriptors.}

$$
\begin{gathered}
\log \operatorname{ER}(J)=1.722-172.3 * \mathrm{e} 41(\text { any,np })+ \\
57.58 * \mathrm{e} 48(\mathrm{np}, \mathrm{np})-263.6 * \mathrm{e} 13(\mathrm{np}, \mathrm{np})+ \\
132.1 * \mathrm{e} 21(\text { any,np })+74.99 * \mathrm{e} 10(\mathrm{hs}, \mathrm{hs})+ \\
1.760 * \mathrm{e} 2(\mathrm{hba}, \mathrm{hbd})-2.072 * \mathrm{e} 3(\mathrm{hba}, \mathrm{hs}) \\
N=42, r^{2}=0.626, q^{2}=0.473
\end{gathered}
$$

\section{Classic QSAR and 4D-Fingerprint Descriptors.}

$$
\begin{gathered}
\log \operatorname{ER}(J)=1.957-0.0002 *(\text { LUMO }-109.5)^{2}- \\
0.112 *(\text { Chi } 5-6.992)^{2}+1.236 * \text { Dipole }+ \\
\left.31.42 *(\mathrm{e} 3(\text { hba,hs })-0.102)^{2}+216.6 * \text { e17(np,np }\right)- \\
312.3 * \mathrm{e} 18(\text { any,any })(17 \\
N=42, r^{2}=0.825, q^{2}=0.753
\end{gathered}
$$

The optimal classic descriptor and 4D-fingerprint QSAR models each contain seven descriptor terms. The classic descriptor model is significantly better in terms of $r^{2}$ and $q^{2}$ than the 4D-fingerprint model. However, the classic descriptor model has five quadratic terms, while the 4D-fingerprint model has none. It is worth noting that 4D-fingerprint descriptors seem "resistant" to becoming quadratic descriptor terms. Perhaps the most significant finding in this part of the study is that the combination of classic and 4D-fingerprint descriptors leads to a good 6-term model, eq 17, as judged by $r^{2}$ and $q^{2}$, even though this QSAR model does contain three quadratic terms.

The fourth training set has only 17 compounds and uses 5FU as the reference penetrant. Optimal QSAR models for this small training set were based on 76 classic descriptors and 334 4D-fingerprints and required employing $\operatorname{ER}(J)$ directly, not $\log \operatorname{ER}(J)$, as the dependent variable.

Classic QSAR Descriptors. No significant QSAR model could be constructed.

\section{D-Fingerprint Descriptors.}

$$
\begin{gathered}
\mathrm{ER}=-8506 * \mathrm{e} 6(\mathrm{hs}, \mathrm{hs})-837 * \mathrm{e} 3(\mathrm{hs}, \mathrm{hs})-6410 * \mathrm{e} 13 \\
(\text { any,any })-694 * \mathrm{e} 2(\mathrm{hs}, \mathrm{hs})+900.14(18 \mathrm{a}) \\
N=17, r^{2}=0.883, q^{2}=0.742 \\
\mathrm{ER}=1188551 *[\mathrm{e} 6(\mathrm{hs}, \mathrm{hs})-0.038]^{2}+ \\
14587691 *[\mathrm{e} 14(\mathrm{np}, \mathrm{p}+)-0.013]^{2}+ \\
531759[\mathrm{e} 5(\mathrm{hs}, \mathrm{hs})-0.043]^{2}-8.11(18 \mathrm{~b}) \\
N=17, r^{2}=0.930, q^{2}=0.702
\end{gathered}
$$

Classic and 4D-fingerprint Descriptors - Same as Eqs $18 \mathrm{a}$ and $18 b$.

$$
\begin{gathered}
\mathrm{ER}=-8506 * \mathrm{e} 6(\mathrm{hs}, \mathrm{hs})-837 * \mathrm{e} 3(\mathrm{hs}, \mathrm{hs})-6410 * \mathrm{e} 13 \\
(\text { any,any })-694 * \mathrm{e} 2(\mathrm{hs}, \mathrm{hs})+900.14 \text { (19a) } \\
N=17, r^{2}=0.883, q^{2}=0.742 \\
\mathrm{ER}=1188551 *[\mathrm{e} 6(\mathrm{hs}, \mathrm{hs})-0.038]^{2}+ \\
14587691 *[\mathrm{e} 14(\mathrm{np}, \mathrm{p}+)-0.013]^{2}+ \\
531759[\mathrm{e} 5(\mathrm{hs}, \mathrm{hs})-0.043]^{2}-8.11(19 \mathrm{~b}) \\
N=17, r^{2}=0.930, q^{2}=0.702
\end{gathered}
$$

It is difficult to build good QSAR models for training set 4 because (a) the inability to construct a significant model employing only classic QSAR descriptors; (b) the optimal linear 4D-fingerprint model, eq 18a, contains four descriptor terms, which likely nears overfitting the data; (c) the need to include all quadratic terms in a 3-term 4D-fingerprint model, eq 18b; and (d) the large regression coefficients in eq $18 \mathrm{~b}$ and large constant term in eq $18 \mathrm{a}$.

The results of the random scrambling and test set prediction-validation studies are given in Table 7 . The random scrambling studies lead to models with average $r^{2}$ values of about 0.35 across the four training sets. The corresponding $r^{2}$ values of the optimum QSAR models built for the training sets range from 0.767 to 0.883 . Thus, the random scrambling studies suggest that the QSAR models are significant and not the result of chance correlations.

The test set validation studies indicate that test training set models constructed from random samplings, using $85 \%$ of the compounds from the original training sets, are quite comparable in both $r^{2}$ and $q^{2}$ to the optimum models developed for the respective training sets [eqs 10-19]. Moreover, the ability to build consistent models across subsets of the original training set suggests that all of these models are stable and robust with respect to the range of the chemistry sampled in the training set.

Finally, the average $r^{2}$ of prediction across test set compounds using the QSAR models built from the original training sets is consistently about $8-15 \%$ less than the corresponding $r^{2}$ of the QSAR models. This indicates stable and meaningfully predictive models.

\section{DISCUSSION}

The QSARs developed for each of the four training sets are discussed below within the context that different penetra- 
tion enhancement mechanisms may play a role in both the chemical structure of the enhancer and the penetrant.

QSAR Models of Training Set 1. The model using both classic QSAR descriptors and 4D-fingerprint descriptors (eq 12 ) is the best model, but all three models (eqs 10-12) are close in statistical significance. Three descriptor terms in eq 12 are also found in eqs 10 and 11 , namely e18(hs,hs), \{e22(any,any) versus e16(any,any)\}, and FH2O. Thus, these terms are judged to capture the most significant features for nonpolar, surfactant-like skin penetration enhancers in combination with relatively large, nonpolar penetrants like HC. A possible composite interpretation of these descriptors is as follows: (a) As aqueous solubility (FH2O and PSA) increases, skin enhancer "potency" decreases. (b) There is a definitive optimum size and likely shape for the bestperforming enhancer, as judged by the positive contribution to $\operatorname{ER}(J)$ from e18(hs,hs) and the negative contribution from e22(any,any). (c) The less exposed the polar surface of the enhancer is in the membrane, the more effective the enhancer, (PSA). (d) The short-range distribution [3-5 $\mathrm{A}$ ] of polarpositive atoms over the enhancer $(\mathrm{e} 1(\mathrm{p}+, \mathrm{hs}))$ can contribute to increasing potency, which may be indicative of a specific membrane-enhancer "binding" interaction.

QSAR Models of Training Set 2. The QSAR model using the 4D-fingerprint descriptors, eq 13 , is the best model. No meaningful model could be built using only classic QSAR descriptors, and when the classic descriptors were combined with the 4D-fingerprint descriptors the resulting optimal QSAR model was identical to eq 13 . The inability to construct a significant QSAR model using only classic QSAR descriptors may be indicative of the enhancement mechanism involving local regions of the enhancers (a pharmacophore), as opposed to the entire molecule. That is, the classic QSAR descriptors may generally determine whole molecule features and properties - not information about specific parts of a molecule. The all-4D-fingerprint model, given by eqs 13 and 14 , is composed completely of quadratic descriptor terms. Quadratic 4D-fingerprint terms may reflect the need for forced nonlinear data-fitting. A possible mechanistic interpretation of the descriptors of eq 13 is as follows: (a) The short-range $[3-5 \AA$ joint distribution of hydrogen-bonding groups and nonpolar groups over the enhancer (e1(np,hbd)) can contribute to increasing enhancer potency, which may indicate a specific "binding" interaction involving the enhancer with sites in the strateum corneum of the skin. (b) Nonpolar groups and their distribution over an enhancer generally decreases skin enhancer "potency" (e3(np,np), e3(any,np), and e16(any,np)). (c) Short-range shape steric restrictions on these polar enhancers may be reflected by e4(any, any).

QSAR Models of Training Set 3. This QSAR model, eq 17, which uses both classic QSAR descriptors and 4Dfingerprint descriptors, proves the best overall and, somewhat surprisingly, is considerably more significant than the optimum QSAR models built solely from classic QSAR descriptors, eq 15, and 4D-fingerprint descriptors, eq 16. The $r^{2}$ and $q^{2}$ values for eq 17 may approach the highest possible values without overfitting the training set data. Two of the three classic QSAR descriptor terms of eq 17 [LUMO and Dipole] are also found in eq 15, the QSAR model based solely on classic QSAR descriptors. A possible mechanistic interpretation of the descriptors of eq 17 is as follows: (a)
As the dipole moment of the skin penetration enhancer and its corresponding "dipole-dipole binding" to a site in the stratum corneum, such as the head groups of membrane phospholipids, increases, so does the skin penetration enhancer "potency". (b) An optimum size and likely shape for the best performing enhancer exists as judged by the negative contribution to the $\log \operatorname{ER}(J)$ from e18(any,any) and the negative contribution from Chi5. (c) The optimum size and shape reflected by e18(any,any) are intimately related to the long-range distribution of nonpolar groups over the enhancer as captured by e17(np,np), indicating the aliphatic "tails" of the enhancers may be inserting into the assemblies of the membrane phospholipids, including cellular membrane monolayers. (d) The short-range distribution of hydrogen bond acceptors over the enhancer (e3(hba,hs)) can contribute to increasing enhancer potency. This may reflect a specific membrane-enhancer "binding" interaction, and this term may denote the near equivalent type of interaction characterized by e1(p+,hs) in eq 12 as described training set 1. (e) The role of the LUMO term in eq 17 is unclear but does suggest this nonground descriptor captures some important features of the penetration enhancement process, perhaps related to chemical reactivity.

QSAR Models for Training Set 4. All but one 4Dfingerprint of the terpene-5FU enhancer system is of the (any, any) or (hs, hs) pairs of atom types. The 4D-fingerprint QSAR models of the other skin penetration enhancer systems [training sets 1-3] also contain 4D-fingerprints from the (any, any) and (hs, hs) atom pair types. These QSAR models, however, also contain a significant number of 4D-fingerprint descriptors from nonpolar (np) atom types. Thus, it appears that steric and/or molecular shape interactions govern the terpene-5FU system with no significant nonpolar or electrostatic interactions.

A comparison analysis of the descriptor terms and their respective regression coefficients across the pairs of the best QSAR models [eqs 12, 13, 17, and 18a] from the four skin penetration enhancer data sets does not reveal any significant similar terms. Even allowing for a generous degree of assumed commonality among the descriptors, especially for the 4D-fingerprints, it is impossible to formulate a nearcommon mechanistic model for penetration enhancer action for any pair of the four data sets. Thus, the QSAR models for the penetrator enhancer systems are judged meaningfully different from one another. This suggests multiple mechanisms of skin penetration enhancement, dependent on the chemistry of both the enhancer and the penetrant.

We will further discuss the QSAR analyses and mechanistic modeling of skin penetration enhancers in our next paper, focusing on the results of using membrane-interaction (MI)-QSAR analysis to model the behavior of skin penetration enhancers in phospholipid membrane assemblies.

\section{ACKNOWLEDGMENT}

This work was funded by the National Institutes of Health through the NIH Roadmap for Medical Research, Grant 1 R21 GM075775-01. Information on Novel Preclinical Tools for Predictive ADME-Toxicology can be found at http:// grants.nih.gov/grants/guide/rfa-files/RFA-RM-04-023.html. Links to nine initiatives are found at http://nihroadmap.nih.gov/initiatives.asp. This work was also supported by Avon 
Products, Inc. Resources of The Chem 21 Group, Inc. were used in performing these studies. We are grateful to Tony Hopfinger, Jr. for his assistance in the editing and proofreading of this manuscript.

\section{REFERENCES AND NOTES}

(1) Barry, B. Mode of action of penetration enhancers in human skin. $J$. Controlled Release 1987, 6, 85-97.

(2) Kim, N.; El-Kattan, A.; Asbill, C. S.; Kennette, R. J.; Sowell, J. W., Sr.; Latour, R.; Michniak, B. B. Evaluation of derivatives of 3-(2oxo-1-pyrrolidine) hexahydro-1H-azepine-2-one as dermal penetration enhancers: side chain length variation and molecular modeling. $J$. Controlled Release 2001, 73, 183-196.

(3) Kanikkannar, N.; Kandimalla, K.; Lamba, S. S.; Singh, M. Structureactivity relationships of chemical penetration enhancers in transdermal drug delivery. Curr. Med. Chem. 1999, 6, 593-608.

(4) http://www.cancerbackup.org.uk/Treatments/Chemotherapy/Individualdrugs/Fluorouracil. (accessed 12/12/2006).

(5) Ward, J. H. Hierarchical grouping to optimize an objective function. J. Am. Stat. Assoc. 1963, 58, 236-244.

(6) Michniak, B. B.; Player, M. R.; Godwin, D. A.; Phillips, C. A.; Sowell, J. W., Sr. Investigation of enhancer structure activity relantionships in congeners of 2-(1-Nonyl)-1,3-dioxolane. Drug Delivery 1995, 2, $117-122$.

(7) Michniak, B. B.; Player, M. R.; Godwin, D. A.; Lockhart, C. C.; Sowell, J. W., Sr. In vitro evaluation of a series of azone analogs as dermal penetration enhancers: V. miscellaneous compounds. Int. J. Pharm. 1998, 161, 169-178.

(8) Fuhrman, L. C., Jr.; Michniak, B. B.; Behl, C. R.; Malick, A. W. Effect of novel penetration enhancers on the transdermal delivery of hydrocortisone: an in vitro species comparison. J. Controlled Release 1997, 45, 199-206.

(9) Godwin, D. A.; Player, M. R.; Sowell, J. W., Sr.; Michniak, B. B. Synthesis and investigation of urea compounds as transdermal penetration enhancers. Int. J. Pharm. 1998, 167, 165-175.

(10) Godwin, D. A.; Michniak, B. B. Influence of drug lipophilicity on terpenes as transdermal penetration enhancers. Drug Dev. Ind. Pharm. 1999, 25, 905-915.

(11) Michniak, B. B.; Player, M. R.; Sowell, J. W., Sr. Synthesis and in vitro transdermal penetration enhancing activity of lactam n-acetic acid esters. J. Pharm. Sci. 1996, 85, 150-154.
(12) Michniak, B. B.; Player, M. R.; Godwin, D. A.; Phillips, C. A.; Sowell, J. W., Sr. In vitro evaluation of a series of azone analogs as dermal penetration enhancers: IV. amines. Int. J. Pharm. 1995, 116, 201209.

(13) Michniak, B. B.; Player, M. R.; Fuhrman, L. C.; Christensen, C. A.; Chapman, J. M., Jr.; Sowell, J. W., Sr. In vitro evaluation of a series of azone analogs as dermal penetration enhancers: II. (thio)amides. Int. J. Pharm. 1993, 94, 203-210.

(14) Kim, N.; El-Kattan, A.; Henary, M. M.; Strekowski, L.; Michniak, B. B. Percutaneous penetration enhancement activity of aromatic s,s-dimethyliminosulfuranes. Int. J. Pharm. 1999, 187, 219229.

(15) Song, Y.; Xiao, C.; Mendelsohn, R.; Zheng, T.; Strekowski, L. Michniak, B. B. Investigation of iminosulfuranes as novel transdermal penetration enhancers: enhancement activity and cytotoxicity. Pharm. Res. 2005, 22, 1918-1925.

(16) Michniak, B. B.; Player, M. R.; Fuhrman, L. C.; Christensen, C. A.; Chapman, J. M., Jr.; Sowell, J. W., Sr. In vitro evaluation of a series of azone analogs as dermal penetration enhancers: III. acyclic amides. Int. J. Pharm. 1994, 110, 231-239.

(17) Michniak, B. B.; Player, M. R.; Chapman, J. M., Jr.; Sowell, J. W., Sr. In vitro evaluation of a series of azone analogs as dermal penetration enhancers: I. Int. J. Pharm. 1993, 91, 85-93.

(18) William, A.; Barry, B. Terpene pentration enhancers of 5FU. Pharm. Res. 1991, 8, 17-24.

(19) Takayama, K.; Kikuchi, K.; Obata, Y.; Okabe, H.; Machida, Y.; Nagai, T. Terpenes as percutaneous absorption promoters. S.T.P. Pharm. Sci. 1991, $1,83-88$

(20) Duca, J. S.; Hopfinger, A. J. Estimation of molecular similarity based on 4D-QSAR analysis: formalism and validation. J. Chem. Inf. Comput. Sci. 2001, 41, 1367-1387.

(21) Senese, C. L.; Duca, J. S.; Pan, D.; Hopfinger, A. J.; Tseng, Y. J. 4D-fingerprints, universal QSAR and QSPR descriptors. J. Chem. Inf. Comput. Sci. 2004, 44, 1526-1539.

(22) Rogers, D.; Hopfinger, A. J. Applications of genetic function approximation to quantitative structure-activity relationships and quantitative structure-property relationships. J. Chem. Inf. Comput. Sci. 1994, 34, 854-866.

(23) Rogers, D. WOLF 6.2 GFA Program; Molecular Simulation Inc.: San Diego, CA, 1994.

(24) Friedman, J. MultiVariate Adaptive Regression Splines; Stanford University: Stanford, CA, 1988.

CI700051E 